\title{
Continuous saline bladder irrigation for two hours following transurethral resection of bladder tumors in patients with non- muscle invasive bladder cancer does not prevent recurrence or progression compared with intravesical Mitomycin-C
}

\author{
Andrew T. Lenis ${ }^{1,2,3}$ (D) Kian Asanad ${ }^{1}$, Maher Blaibel ${ }^{4}$, Nicholas M. Donin ${ }^{1,2,3}$ and Karim Chamie ${ }^{1,2,3^{*}}$
}

\begin{abstract}
Background: Intravesical Mitomycin-C (MMC) following transurethral resection of bladder tumor (TURBT), while efficacious, is associated with side effects and poor utilization. Continuous saline bladder irrigation (CSBI) has been examined as an alternative. In this study we sought to compare the rates of recurrence and/or progression in patients with NMIBC who were treated with either MMC or CSBI after TURBT.

Methods: We retrospectively reviewed records of patients with NMIBC at our institution in 2012-2015. Perioperative use of MMC (40 mg in $20 \mathrm{~mL}$ ), CSBI (two hours), or neither were recorded. Primary outcome was time to recurrence or progression. Descriptive statistics, chi-squared analysis, Kaplan-Meier survival analysis, and Cox multivariable regression analyses were performed.

Results: 205 patients met inclusion criteria. Forty-five (22.0\%) patients received CSBI, 71 (34.6\%) received MMC, and 89 (43.4\%) received no perioperative therapy. On survival analysis, MMC was associated with improved DFS compared with CSBI $(p=0.001)$ and no treatment $(p=0.0009)$. On multivariable analysis, high risk disease was associated with increased risk of recurrence or progression (HR 2.77, 95\% Cl: 1.28-6.01), whereas adjuvant therapy (HR 0.35, 95\% Cl: 0.20-0.59) and MMC (HR 0.43,95\% Cl: 0.25-0.75) were associated with decreased risk.

Conclusions: Postoperative MMC was associated with improved DFS compared with CSBI and no treatment. The DFS benefit seen with CSBI in other studies may be limited to patients receiving prolonged irrigation. New intravesical agents being evaluated may consider saline as a control given our data demonstrating that short-term CSBI is not superior to TURBT alone.
\end{abstract}

Keywords: Bladder cancer, Therapeutic irrigation, Mitomycin-C, Recurrence, Outcome assessment

\footnotetext{
* Correspondence: kchamie@mednet.ucla.edu

'David Geffen School of Medicine at the University of California Los Angeles,

300 Stein Plaza, Suite 348, Los Angeles, California 90095, USA

${ }^{2}$ Department of Urology, Health Services Research Group, David Geffen

School of Medicine at UCLA, Los Angeles, California, USA

Full list of author information is available at the end of the article
}

(c) The Author(s). 2018 Open Access This article is distributed under the terms of the Creative Commons Attribution 4.0 International License (http://creativecommons.org/licenses/by/4.0/), which permits unrestricted use, distribution, and reproduction in any medium, provided you give appropriate credit to the original author(s) and the source, provide a link to the Creative Commons license, and indicate if changes were made. The Creative Commons Public Domain Dedication waiver (http://creativecommons.org/publicdomain/zero/1.0/) applies to the data made available in this article, unless otherwise stated. 


\section{Background}

Non-muscle invasive bladder cancer (NMIBC) accounts for approximately $70 \%$ of new cases of urothelial carcinoma of the bladder. [1] NMIBC has been considered a chronic disease due to its high risk of future complications, including recurrence, which necessitates frequent monitoring and surveillance. The lifelong risk of recurrence and repeated interventions contributes to poor physician and patient compliance with published guidelines, and it significantly burdens the healthcare system from a financial standpoint. [2, 3] Therefore, strategies to prevent recurrence and future complications are paramount to reducing long-term morbidity and mortality.

The standard adjuvant therapy following transurethral resection of bladder tumor (TURBT) for NMIBC is intravesical instillation of Mitomycin-C (MMC), which has been shown to decrease rates of recurrence by approximately $11 \%$, although this is variable depending on the number of and time from prior recurrences. $[4,5]$ The posited mechanism of action is to prevent free-floating tumor cells in the urine following TURBT from re-implanting onto the bladder wall. Although rare, MMC can potentially cause several significant side effects, including severe lower urinary tract symptoms, persistent chronic bladder pain, and even bladder necrosis in case reports. [6] Furthermore, MMC is contraindicated when there is a concern for bladder perforation and when there is significant post-operative gross hematuria. Considering these limitations, there is an urgent need for alternative strategies to prevent the re-implantation of tumor cells following TURBT, to reduce recurrence and minimize the morbidity of the disease. A 2012 Cochrane review of intravesical gemcitabine yielded conflicting results. [7] Apaziquone is a novel intravesical alkylating agent that has demonstrated safety and tolerability in patients as a post-TURBT instillation and is being evaluated in Phase 3 clinical trials (NCT02563561). [8] Alternatively, several groups have utilized sterile water and saline irrigation over $18-24 \mathrm{~h}$ as a strategy to lyse floating tumors cells and prevent the re-implantation of cells into the bladder wall. $[9,10]$ In our current study, we sought to evaluate continuous bladder irrigation with isotonic $(0.9 \% \mathrm{NaCl})$ normal saline (CBSI) for two hours following TURBT as a strategy to reduce recurrence or progression in patients with NMIBC.

\section{Methods}

\section{Patient cohort}

Patients undergoing endoscopic resection of bladder tumors at our institution between March 2012 and July 2015 were identified from the medical record by Current Procedure Terminology (CPT)-4 codes for transurethral biopsy and resection (52204, 52214, 52224, 52234, 52235, 52240). Pathologic and clinical reports were reviewed, and patients with NMIBC were selected for inclusion in the cohort. We excluded all patients with variant histology, including small cell, squamous cell, adenocarcinoma, lymphepithelioid, sarcomatoid, and micropapillary disease. We also excluded patients with a diagnosis of upper tract urothelial carcinoma within one year, unresectable volume of tumor, known metastatic disease, less than three months of follow-up, or patients who underwent cystectomy within three months of diagnosis. Patients were categorized based on a modified AUA Risk Stratification for NMIBC. [11] Low risk was defined as a solitary LG lesion $<2 \mathrm{~cm}$. Intermediate risk was defined as any LG T1, solitary LG Ta $>2 \mathrm{~cm}$, multiple LG Ta, solitary HG Ta $<2 \mathrm{~cm}$, or a history of LG NMIBC. High risk was defined as any CIS, HG T1, HG $\mathrm{Ta}>2 \mathrm{~cm}$, multiple HG Ta, or any history of HG Ta lesions or BCG recurrence. Modification of the AUA risk groups was made in order to conform to the size criteria used in the current procedural terminology codes for TURBT. Follow-up was calculated based on the time of the last cystoscopy. All study conduct was approved by the Institutional Review Board at our institution.

\section{Independent variables}

All patients received adjuvant CSBI, adjuvant MMC, or no adjuvant treatment at the discretion of the operating surgeon. Typically, patients for whom there was a concern for bladder perforation were not given CSBI or MMC. MMC was given as an instillation of $40 \mathrm{mg}$ in $20 \mathrm{~mL}$ of saline. Following a dwell time of 60-90 min, the MMC was drained from the bladder and the catheter was left in place if deemed necessary by the surgeon. CSBI was performed by placement of a three-way Foley catheter at the conclusion of the case and was left running for approximately two hours post-operatively. The rate was kept at maximum flow without titration for this time. Patients did not require an overnight stay specifically for CSBI.

\section{Dependent variables}

Our dependent variable of interest was time to recurrence or progression. Recurrence was defined as the presence of pathologically confirmed urothelial carcinoma on biopsy or repeat resection. Patients who were found to have a lesion visible on cystoscopy that warranted intervention in the office (e.g. fulguration) were also classified as having disease recurrence. Cytology results obtained at the time of office fulguration were recorded. Progression was defined as any increase in grade or stage of disease.

\section{Statistical analysis}

Comparisons between categorical variables were tested using Chi-squared analysis and Fisher's exact test when appropriate. The two-sample Student's t-test was used to 
test for differences between continuous variables. Differences in disease-free survival (DFS) were analyzed using the Kaplan-Meier method. Cox proportional hazards models were used to estimate hazards ratios for covariates of interest. All statistical analyses were performed with Stata statistical software version 14 (StataCorp, College Station, TX).

\section{Results}

A total of 205 patients underwent TURBT for NMIBC during the study period and met all inclusion criteria. Mean age was $71.9(\mathrm{SD}=11.4)$ years and $81.5 \%$ were male. Low grade (LG) and high grade (HG) were the primary grades in $105(51.2 \%)$ and $100(48.8 \%)$ patients, respectively. Stage was Ta without CIS, Ta with CIS, T1 without CIS, T1 with CIS, and CIS alone in 126 (61.5\%), 12 (5.9\%), 36 (17.6\%), 13 (6.3\%), and 18 (8.8\%) patients, respectively. Tumor size was $<0.5 \mathrm{~cm}, 0.5-2 \mathrm{~cm}, 2-$ $5 \mathrm{~cm}$, and $>5 \mathrm{~cm}$ in 20 (9.8\%), 90 (43.9\%), 45 (21.9\%), and $50(24.4 \%)$ patients, respectively. Multiple tumors were present in 105 (51.2\%) patients and 75 (36.6\%) had a history of NMIBC. A modified AUA risk stratification as discussed in the methods resulted in 23 (11.2\%) low risk patients, 80 (39\%) intermediate risk patients, and 102 (49.8\%) high risk patients. As immediate perioperative therapy, a total of $45(22.0 \%)$ patients had CSBI, 71 (34.6\%) had MMC, and 89 (43.4\%) had no perioperative therapy. Only $36(19.8 \%)$ of patients with intermediate or high risk disease underwent a restaging TURBT. Eighty-six (42.0\%) patients received adjuvant intravesical therapy, most commonly with bacillus Calmette-Guérin (BCG $n=76)$, BCG + interferon $(n=6)$, Gemcitabine ( $n$ $=2)$, or MMC $(\mathrm{n}=2)$. Table 1 and Table 2 summarize the cohort characteristics stratified by perioperative treatment and recurrence and progression, respectively.

Median follow-up time for the entire cohort was 16 [Interquartile range (IQR): 8-28] months. A total of 74 (36.1\%) patients recurred at a median of 9.5 [IQR: 4-14] months and $16(7.8 \%)$ progressed at a median of 16 [IQR: 6-31.5] months. The median DFS was 25 months for those who received no perioperative treatment, 55 months for those receiving MMC, and 16 months for those receiving CSBI. The Kaplan-Meier survival curve is presented in Fig. 1 and demonstrates a significant DFS advantage of MMC compared with either CSBI or no perioperative treatment (log rank test: $p<0.01)$. Kaplan-Meier curves for patients with a combination of low and intermediate risk NMIBC (log rank test: $p=$ 0.02 ) and high risk NMIBC (log rank test: $p=0.04$ ), and are presented in Figs. 2 and 3, respectively.

Lastly, we created a multivariable model incorporating age, AUA risk stratification, use of additional adjuvant therapy, and type of perioperative therapy (None, MMC, or CSBI). On Cox multivariable modeling, high risk was associated with increased risk of recurrence or progression (HR 2.77, 95\% CI: 1.28-6.01), whereas adjuvant therapy (HR 0.35, 95\% CI: 0.20-0.59) and MMC (HR 0.43, 95\% CI: 0.25-0.75) were associated with decreased risk of recurrence or progression (Table 3).

\section{Discussion}

The burden of NMIBC includes high financial costs to the healthcare system, significant risk of recurrence that necessitates life-long invasive surveillance, and uncertainty of possible progression that would prompt future radical operative intervention, especially in the highest-risk patients. Strategies to reduce the risk of recurrence and progression, including intravesical chemotherapy and immunotherapy, have been shown to be effective. $[4,12]$ However, none of these are without risk of potential significant side effects. In our current study we sought to utilize postoperative CSBI in a fashion similar to MMC, as an immediate, one-time postoperative treatment following surgery. This strategy avoids the toxicity of intravesical chemotherapy, as well as the inconvenience of an overnight hospital stay for prolonged CSBI.

In our cohort, however, post-operative CSBI for two hours was not equivalent to a single dose of perioperative MMC. Given the small numbers of patients in the low risk subgroup, we combined patients from low risk and intermediate risk groups for analysis. In the low and intermediate risk patients, there was a significant improvement in DFS with MMC compared with CSBI. In fact, CSBI performed no better than no perioperative treatment. In the high risk subgroup, a similar trend was observed. In our study the absolute risk reduction of postoperative MMC compared with no treatment at one year was $12.3 \%$, which is similar to what is reported in the literature (11.7\%). [4, 13] This benefit of MMC holds true even in our Cox multivariable model.

With respect to the efficacy of CSBI, our data stands in contrast to results published by others, albeit with some important differences in study design. Onishi et al. performed a non-randomized study comparing 18-22 h of post-operative CSBI to a full year of induction and maintenance $\mathrm{MMC}$ in patients with European Organization for Research and Treatment of Cancer (EORTC) intermediate risk NMIBC and showed no difference in several outcomes, including recurrence-free rates, time to first recurrence, and frequency of recurrences. [10] In this manuscript, the authors alluded to a planned prospective study that was recently published. [14] In their follow-up study, 227 patients with primary EORTC low- to intermediate-risk (all LG) NMIBC were randomized 1:1 to receive CSBI for $18 \mathrm{~h}$ or a single dose of $30 \mathrm{mg}$ of MMC in $30 \mathrm{~mL}$ of saline. After a median follow-up of 37 months, $29 \%$ of patients experienced a recurrence. Recurrence-free rates at 1,3 , and 5 years were similar between the CSBI 
Table 1 Cohort characteristics stratified by perioperative treatment

\begin{tabular}{|c|c|c|c|c|}
\hline Variable & No treatment & MMC & $\mathrm{CSBI}$ & $p$-value \\
\hline Total no. of patients & 89 & 71 & 45 & - \\
\hline Age, mean (SD) & $73.2(11.2)$ & $68.2(12.3)$ & $75.3(8.9)$ & $<0.002+$ \\
\hline Gender, $n(\%)$ & & & & 0.54 \\
\hline Male & $75(84.3)$ & $55(77.5)$ & $37(83.2)$ & \\
\hline Female & $14(15.7)$ & $16(22.5)$ & $8(17.8)$ & \\
\hline Grade, $n(\%)$ & & & & 0.9 \\
\hline High & $45(50.6)$ & $34(47.9)$ & $21(46.7)$ & \\
\hline Low & $44(49.4)$ & $37(52.1)$ & $24(53.3)$ & \\
\hline Stage, $n(\%)$ & & & & $0.03^{*}$ \\
\hline Ta without CIS & $55(61.8)$ & $41(57.8)$ & $30(66.7)$ & \\
\hline Ta with CIS & $3(3.4)$ & $4(5.6)$ & $5(11.1)$ & \\
\hline $\mathrm{T} 1$ without CIS & $13(14.6)$ & $18(25.4)$ & $5(11.1)$ & \\
\hline T1 with CIS & $4(4.5)$ & $6(8.5)$ & $3(6.7)$ & \\
\hline CIS only & $14(15.7)$ & $2(2.8)$ & $2(4.4)$ & \\
\hline Tumor size, $n(\%)$ & & & & $0.12^{*}$ \\
\hline$<0.5 \mathrm{~cm}$ & $11(12.36)$ & $3(4.2)$ & $6(13.3)$ & \\
\hline $0.5-2.0 \mathrm{~cm}$ & $33(37.1)$ & $41(57.8)$ & $16(35.6)$ & \\
\hline $2.0-5.0 \mathrm{~cm}$ & $22(24.7)$ & $13(18.3)$ & $10(22.2)$ & \\
\hline$>5.0 \mathrm{~cm}$ & $23(25.8)$ & $14(19.7)$ & $13(28.9)$ & \\
\hline Multiple tumors, $n(\%)$ & $47(52.8)$ & $36(50.7)$ & $22(48.9)$ & 0.91 \\
\hline Recurrent disease, $n$ (\%) & $40(45.0)$ & $23(32.4)$ & $12(26.7)$ & 0.08 \\
\hline AUA Risk Stratification & & & & 0.72 \\
\hline Low risk & $10(11.2)$ & $6(8.5)$ & $7(15.6)$ & \\
\hline Intermediate risk & $34(38.2)$ & $31(43.7)$ & $15(33.3)$ & \\
\hline High risk & $45(50.6)$ & $34(47.9)$ & $23(51.1)$ & \\
\hline Restaging resection, $n(\%)$ & $8(9.0)$ & $18(25.4)$ & $10(22.2)$ & 0.02 \\
\hline Adjuvant therapy, $n$ (\%) & $35(39.3)$ & $35(49.3)$ & $16(35.6)$ & 0.28 \\
\hline Follow-up in months, median [IQR] & $14[6-28]$ & $23[11-32]$ & 13 [9-19] & $<0.01 \S$ \\
\hline
\end{tabular}

MMC Mitomycin-C, CSBI continuous saline bladder irrigation, SD standard deviation, CIS carcinoma in situ. +One-way ANOVA. *Fisher's exact test. $\S$ non-parametric equality of medians test

and MMC groups on Kaplan-Meier analysis. Subgroup analysis showed no difference when stratified between the low- and intermediate-risk tumors. Adverse events were also compared and the MMC group was found to have significantly higher rates of gross hematuria, irritative bladder symptoms, and dysuria (including retention). While the equivalence of CSBI and MMC demonstrated by Onishi et al. could be explained in part by patient selection (all LG patients), we did not replicate this result even in the low and intermediate risk subgroups of our cohort. One important difference in our protocols is the dose of MMC, which was the standard $40 \mathrm{mg}$ in our study and $30 \mathrm{mg}$ in the study by Onishi et al. The most striking difference between our studies, however, is in the duration of CSBI. We intentionally restricted CSBI to two hours to limit the need for overnight hospital stays. While similarly efficacious to one instillation of MMC, CSBI used by Onishi et al. was titrated over $18 \mathrm{~h}$, and it was not reported how many of these patients required an overnight stay. While the authors debate the cost advantages of saline compared with MMC, we question whether this may be offset by even a small fraction of patients requiring overnight admissions for CSBI. Nevertheless, this data demonstrates that in addition to a standard dose of $40 \mathrm{mg}$ of $\mathrm{MMC}$, duration may be an important component of the efficacy of CSBI in preventing tumor cell reimplantation.

Our results also appear to conflict with the results of a recent meta-analysis utilizing individual patient data from randomized trials comparing immediate intravesical instillation of various chemotherapy agents to TURBT alone or instillation of control solution (saline 
Table 2 Cohort characteristics stratified by Recurrence or Progression

\begin{tabular}{|c|c|c|c|}
\hline Variable & $\begin{array}{l}\text { Recurrence } \\
\text { or Progression }\end{array}$ & $\begin{array}{l}\text { No Recurrence } \\
\text { or Progression }\end{array}$ & p-value \\
\hline Total no. of patients & 90 & 115 & - \\
\hline Age, mean (SD) & $73.6(10.8)$ & $70.6(11.8)$ & $0.07+$ \\
\hline Gender, $n(\%)$ & & & 0.81 \\
\hline Male & $74(82.2)$ & $93(80.9)$ & \\
\hline Female & $16(17.8)$ & $22(19.1)$ & \\
\hline Grade, $n(\%)$ & & & 0.38 \\
\hline High & $47(52.2)$ & $53(46.1)$ & \\
\hline Low & $43(47.8)$ & $62(53.9)$ & \\
\hline Stage, $n(\%)$ & & & $0.09^{*}$ \\
\hline Ta without CIS & $55(61.1)$ & $71(61.7)$ & \\
\hline Ta with CIS & $3(3.3)$ & $9(7.8)$ & \\
\hline T1 without CIS & $14(15.6)$ & $22(19.1)$ & \\
\hline T1 with CIS & $5(5.6)$ & $8(7.0)$ & \\
\hline $\mathrm{CIS}$ & $13(14.4)$ & $5(4.4)$ & \\
\hline Tumor size, $n(\%)$ & & & 0.09 \\
\hline$<0.5 \mathrm{~cm}$ & $14(15.6)$ & $6(5.2)$ & \\
\hline $0.5-2.0 \mathrm{~cm}$ & $37(41.1)$ & $53(46.1)$ & \\
\hline $2.0-5.0 \mathrm{~cm}$ & $17(18.9)$ & $28(24.4)$ & \\
\hline$>5.0 \mathrm{~cm}$ & $22(24.4)$ & $28(24.3)$ & \\
\hline Multiplicity of tumor, $n(\%)$ & $56(62.2)$ & 49 (42.6) & $<0.01$ \\
\hline Recurrent disease, $n(\%)$ & $42(46.7)$ & $33(28.7)$ & $<0.01$ \\
\hline AUA Risk Stratification & & & 0.07 \\
\hline Low risk & $9(10.0)$ & $14(12.2)$ & \\
\hline Intermediate risk & $28(31.1)$ & $52(45.2)$ & \\
\hline High risk & $53(58.9)$ & $49(42.6)$ & \\
\hline Restaging resection, $n(\%)$ & $12(13.3)$ & $24(20.9)$ & 0.16 \\
\hline Adjuvant therapy, $n$ (\%) & $32(35.6)$ & $54(47.0)$ & 0.10 \\
\hline Perioperative treatment, $n$ (\%) & & & 0.004 \\
\hline None & $47(52.2)$ & $42(36.5)$ & \\
\hline MMC & $20(22.2)$ & $51(44.4)$ & \\
\hline CSBI & $23(25.6)$ & $22(19.1)$ & \\
\hline
\end{tabular}

MMC Mitomycin-C, CSBI continuous saline bladder irrigation, SD standard deviation, CIS carcinoma in situ. +One-way ANOVA. *Fisher's exact test

or water). [5] Upon closer examination, however, we are unable to compare the protocols included as published in the meta-analysis or in the original manuscripts to our brief post-operative irrigation protocol. Of the 13 included studies, the use of post-operative irrigation was only documented as consistently used in four of these studies. Irrigation protocols were not detailed in the meta-analysis and review of the original data could not identify specific protocols. Furthermore, at least one study utilized distilled water for irrigation, which has the theoretical advantage of an osmotic cytotoxic effect but the disadvantages of being hypotonic. Therefore, despite a $21 \%$ relative reduction in recurrences found in this meta-analysis with use of post-operative irrigation alone, we can only cautiously compare this result with our data without more detailed information about the irrigation protocols used.

The concept of utilizing irrigation for eradication of residual tumor cells following surgery for cancer is not a new concept, nor is it limited to urology or even endoscopic surgery. Surgeons have traditionally irrigated surgical sites to mechanically wash away debris, dilution of bacterial loads, and as a method of tumor cell lysis, depending on the tonicity of the fluid. A survey in England found that $74 \%$ of general surgeons perform intraoperative peritoneal lavage during cancer operations (36\% 


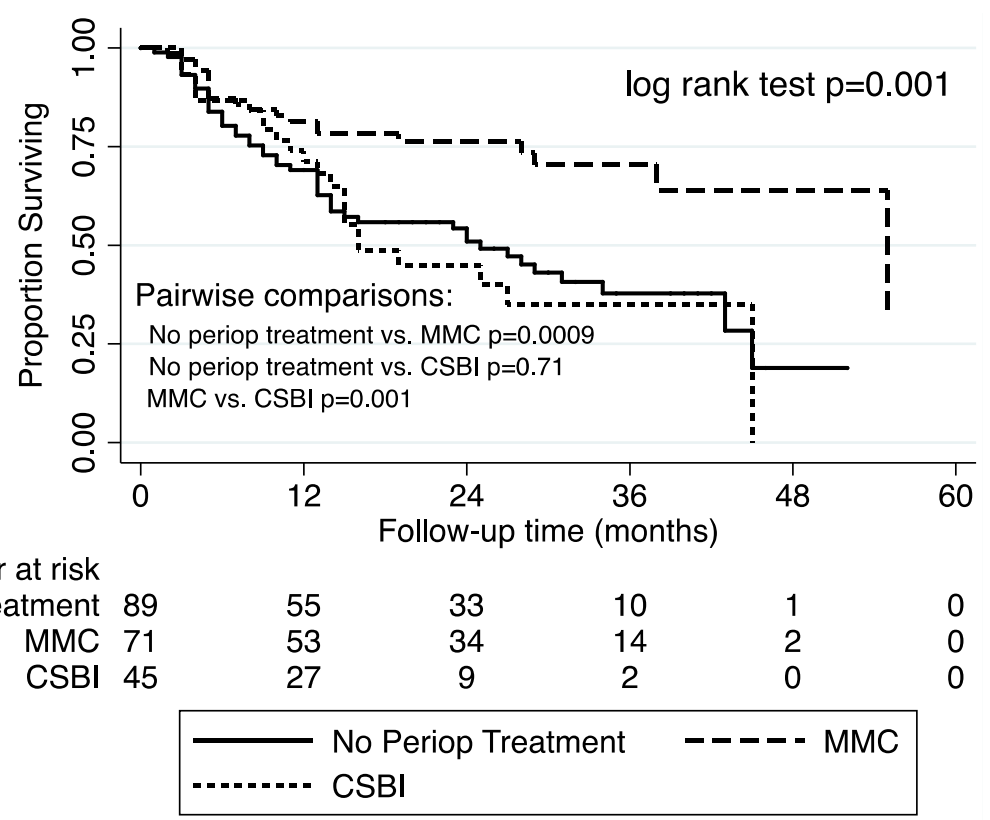

Fig. 1 "DFS in Patients with NMIBC". Kaplan-Meier survival curve for all patients with NMIBC stratified by perioperative treatment. MMC, Mitomycin-C. CSBI, continuous saline bladder irrigation

with water, $21 \%$ with saline, and $17 \%$ with betadine). [15] However, efficacy data on irrigation type is conflicting. Sweitzer et al. designed an experiment in mice to evaluate whether distilled water or sterile saline irrigation could reduce the burden of orthotopically implanted melanoma tumor cells. [16] Unfortunately, they found that neither the mechanical process of irrigation nor the hypotonicity of water reduced the tumor burden. In contrast, Fumito et al. demonstrated the superiority of water irrigation to saline irrigation following laparotomy in a mouse model of colorectal cancer tumor spillage. [17] In head and neck cancer models, both the type of irrigation and type of cancer cell line contributed to efficacy. $[18,19]$ These and other conflicting data suggest that multiple

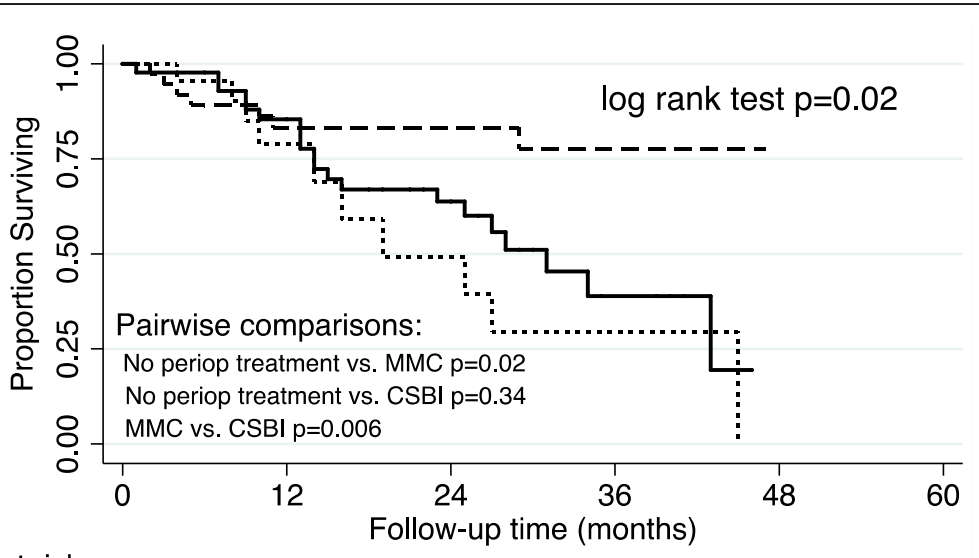

Number at risk

No Periop Treatment 44

MMC 37

CSBI 22

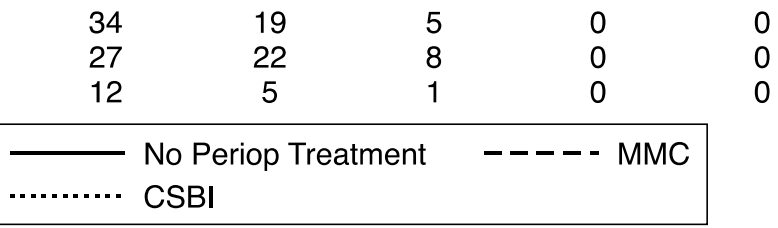

Fig. 2 "DFS in Patients with Low and Intermediate Risk". Kaplan-Meier survival curve for patients with low and intermediate risk disease stratified by perioperative treatment. MMC, Mitomycin-C. CSBI, continuous saline bladder irrigation 


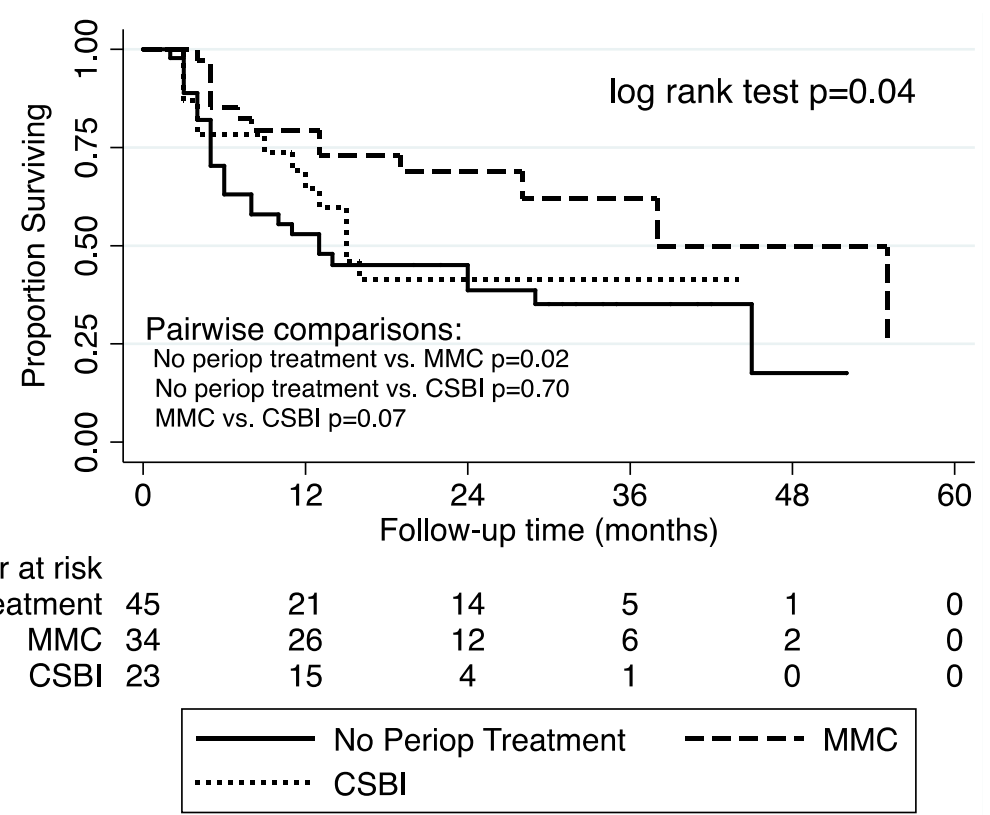

Fig. 3 "DFS in Patients with High Risk". Kaplan-Meier survival curve for patients with high risk disease stratified by perioperative treatment. MMC, Mitomycin-C. CSBI, continuous saline bladder irrigation

factors play a role with respect to the eradication of residual tumor burden, potentially related to the microenvironment and tumor cell-specific factors, such as cell adhesion properties and degree of de-differentiation.

The literature does strongly support irrigation following intra-luminal surgery in other surgical fields. For example, Zhou et al. performed a meta-analysis of studies evaluating intra-luminal washout following anterior resection for rectal cancer and concluded that washout leads to reduced rates of local recurrence. [20] In the urologic literature, Moskovitz et al. first postulated in
1987 that intravesical irrigation with distilled water during and after TURBT would lead to fewer recurrences. [21] While several small studies have demonstrated conflicting results regarding the use of water irrigation compared with no perioperative treatment, no studies have compared CSBI to MMC until the aforementioned studies by Onishi et al. [10, 14, 22, 23] Our study is the first to compare a shorter, perioperative duration of CSBI to both MMC and no perioperative treatment, and to evaluate this strategy in a heterogeneous patient population with low, intermediate, and high risk disease.

Table 3 Cox multivariable model for Recurrence or Progression

\begin{tabular}{|c|c|c|c|}
\hline Variable & Hazard Ratio & $\begin{array}{l}95 \% \text { Confidence } \\
\text { Interval }\end{array}$ & $\mathrm{p}$-value \\
\hline Age (per year of age) & 1.00 & $0.98-1.02$ & 0.92 \\
\hline \multicolumn{4}{|l|}{ AUA Risk Stratification } \\
\hline Low Risk & Reference & Reference & \\
\hline Intermediate Risk & 0.84 & $0.39-1.80$ & 0.66 \\
\hline High Risk & 2.77 & $1.28-6.01$ & 0.01 \\
\hline \multicolumn{4}{|l|}{ Adjuvant therapy } \\
\hline No & Reference & Reference & \\
\hline Yes & 0.35 & $0.20-0.59$ & $<0.001$ \\
\hline \multicolumn{4}{|l|}{ Perioperative treatment } \\
\hline No perioperative treatment & Reference & Reference & \\
\hline MMC & 0.43 & $0.25-0.75$ & 0.003 \\
\hline CSBI & 0.96 & $0.58-1.60$ & 0.89 \\
\hline
\end{tabular}

LG low grade, HG high grade, CIS carcinoma in situ, MMC Mitomycin-C, CSBI continuous saline bladder irrigation 
Our results, however, should be considered within the context of several limitations. Although this was a hypothesis-based study driven by pre-clinical and clinical data, it was not a randomized controlled study, and was limited to the data available in medical records. Furthermore, the study is underpowered and longer term follow-up is required to fully realize the potential differences between treatment groups. It is possible that a larger cohort with longer term follow up could confirm the null hypothesis, suggesting that no difference exists between treatment groups. However, at our institution we are mainly utilizing intravesical gemcitabine based on recently published data that suggests efficacy at a fraction of the cost and with reduced side effects compared with MMC. [24] Consequently, in combination with the current data that suggests inefficacy of $2 \mathrm{~h}$ of CSBI, we are unlikely to treat more patients with adjuvant CSBI. Primarily one surgeon (KC) performed CSBI during the study period while most other surgeons in the department utilized either MMC or no additional perioperative therapy. Therefore, referral patterns may have contributed to patient heterogeneity between groups. Despite some baseline differences between treatment groups described in our results, the data remains consistent when controlling for factors such as tumor grade, stage, and recurrence disease, among others, in a multivariable model. A consistent surveillance cystoscopy protocol was not used for all patients and could have helped standardize follow-up and limit detection bias. Finally, we utilized a clinical definition of recurrence that included any suspicious lesion during office cystoscopy that warranted an intervention (usually fulguration), which may have artificially increased our recurrence rates.

Nevertheless, our study comparing perioperative CSBI, perioperative $\mathrm{MMC}$, and no perioperative treatment answers important questions regarding CSBI as prophylaxis following endoscopic resection for NMIBC. While CSBI for two hours postoperatively should not replace current guideline-recommended perioperative MMC, it does appear that longer duration of CSBI may increase its efficacy. [10, 14] Research is needed to determine whether the duration can be reduced to limit the number of additional hospital stays and whether other, novel perioperative instillations may reduce recurrences and limit side effects.

\section{Conclusions}

Our data demonstrates that perioperative CSBI for two hours following TURBT is not equivalent to postoperative MMC in terms of rates of recurrence or progression. CSBI for two hours appears to be equivalent to no perioperative treatment, regardless of tumor grade. It is possible that CSBI may be required for a longer duration to reduce tumor cell re-implantation and, in turn, decrease rates of recurrence or progression.

\section{Acknowledgements}

This work was supported by the National Institutes of Health Loan Repayment Program (L30 CA154326 (Principal Investigator: KC)), the STOP Cancer Foundation (Principal Investigator: KC), the H \& H Lee Surgical Resident Research Award (Recipient: ATL), and the Short Term Training Program (STTP) at the David Geffen School of Medicine at UCLA (Recipient: KA).

\section{Availability of data and materials}

The datasets generated and/or analyzed during the current study are not publicly available due to patient privacy/protection regulations but may be available from the corresponding author on reasonable request.

\section{Authors' contributions}

ATL was primarily involved in protocol/project development, data collection/ management, data analysis, manuscript writing/editing. KA was involved in data collection/management and manuscript writing. MB was involved in data collection/management and manuscript writing. NMD was involved in protocol/project development, data collection/management, data analysis, manuscript writing/editing. KC supervised and was responsible for all study oversight. All authors read and approved the final manuscript.

Ethics approval and consent to participate

Los Angeles Biomedical Research Institute at Harbor-UCLA Medical Center Institutional Review Board \#14-000648.

Consent for publication

Not required given the retrospective nature of this study.

\section{Competing interests}

The authors declare that they have no competing interests.

\section{Publisher's Note}

Springer Nature remains neutral with regard to jurisdictional claims in published maps and institutional affiliations.

\section{Author details}

${ }^{1}$ David Geffen School of Medicine at the University of California Los Angeles, 300 Stein Plaza, Suite 348, Los Angeles, California 90095, USA. ²Department of Urology, Health Services Research Group, David Geffen School of Medicine at UCLA, Los Angeles, California, USA. ${ }^{3}$ Jonsson Comprehensive Cancer Center, David Geffen School of Medicine at UCLA, Los Angeles, California, USA. ${ }^{4}$ Riverside School of Medicine, University of California, Riverside, California, USA.

Received: 18 October 2017 Accepted: 17 October 2018

Published online: 24 October 2018

\section{References}

1. Clark PE, Agarwal N, Biagioli MC, Eisenberger MA, Greenberg RE, Herr HW, et al. Bladder cancer J Natl Compr Canc Netw. 2013:446-75.

2. James AC, Gore JL. The costs of non-muscle invasive bladder cancer. Urol. Clin. North Am. 2013;40:261-9 Available from: http://www.ncbi.nlm.nih.gov/ pubmed/23540783.

3. Chamie K, Saigal CS, Lai J, Hanley JM, Setodji CM, Konety BR, et al. Compliance with guidelines for patients with bladder cancer: variation in the delivery of care. Cancer. 2011;117:5392-401 Available from: http://www. pubmedcentral.nih.gov/articlerender.fcgi?artid=3206145\&tool= pmcentrez\&rendertype=abstract.

4. Sylvester RJ, Oosterlinck W, van der Meijden APM. A single immediate postoperative instillation of chemotherapy decreases the risk of recurrence in patients with stage ta $\mathrm{T1}$ bladder cancer: a meta-analysis of published results of randomized clinical trials. J Urol. 2004;171:2186-90 quiz2435.

5. Sylvester RJ, Oosterlinck W, Holmäng S, Sydes MR, Birtle A, Gudjonsson S, et al. Systematic review and individual patient data meta-analysis of randomized trials comparing a single immediate instillation of chemotherapy after transurethral resection with transurethral resection alone in patients with stage pTa-pT1 urothelial carcinoma of the bladder: which patients benefit from the instillation? Eur Urol. 2016;69:231-44.

6. Doherty AP, Trendell-Smith N, Stirling R, Rogers $\mathrm{H}$, Bellringer J. Perivesical fat necrosis after adjuvant intravesical chemotherapy. BJU Int. 1999;83:420-3. 
7. Jones G, Cleves A, Wilt TJ, Mason M, Kynaston HG, Shelley M. Intravesical gemcitabine for non-muscle invasive bladder cancer. Cochrane Database Syst Rev John Wiley \& Sons, Ltd. 2012;1:CD009294.

8. Hendricksen K, Cornel EB, de Reijke TM, Arentsen HC, Chawla S, Witjes JA. Phase 2 study of adjuvant intravesical instillations of apaziquone for high risk nonmuscle invasive bladder cancer. J Urol. 2012;187:1195-1199.

9. Taoka R, Williams SB, Ho PL, Kamat AM. In-vitro cytocidal effect of water on bladder cancer cells: the potential role for intraperitoneal lavage during radical cystectomy. CUAJ. 2015;9:E109-13.

10. Onishi T, Sasaki T, Hoshina A, Yabana T. Continuous saline bladder irrigation after transurethral resection is a prophylactic treatment choice for nonmuscle invasive bladder tumor. Anticancer Res. 2011;31:1471-4.

11. Chang SS, Boorjian SA, Chou R, Clark PE, Daneshmand S, Konety BR, et al. Diagnosis and treatment of non-muscle invasive bladder Cancer: AUA/SUO guideline. J Urol. 2016;196:1021-9.

12. Sylvester RJ, van der Meijden APM, Lamm DL. Intravesical bacillus CalmetteGuerin reduces the risk of progression in patients with superficial bladder cancer: a meta-analysis of the published results of randomized clinical trials. J Urol. 2002;168:1964-70 Available from: http://www.ncbi.nlm.nih.gov/ pubmed/12394686.

13. Abern MR, Owusu RA, Anderson MR, Rampersaud EN, Inman BA. Perioperative intravesical chemotherapy in non-muscle-invasive bladder cancer: a systematic review and meta-analysis. J Natl Compr Cancer Netw. 2013;11:477-84

14. Onishi T, Sugino Y, Shibahara T, Masui S, Yabana T, Sasaki T Randomized controlled study of the efficacy and safety of continuous saline bladder irrigation after transurethral resection for the treatment of $\mathrm{n}$... - PubMed NCBI. BJU international. 2016

15. Whiteside OJH, Tytherleigh MG, Thrush S, Farouk R, Galland RB. Intraoperative peritoneal lavage--who does it and why? Ann R Coll Surg Engl. 2005;87:255-8

16. Sweitzer KL, Nathanson SD, Nelson LT, Zachary C. Irrigation does not dislodge or destroy tumor cells adherent to the tumor bed. J Surg Oncol. 1993:53:184-90

17. Ito F, Camoriano M, Seshadri M, Evans SS, Kane JM, Skitzki JJ. Water: a simple solution for tumor spillage. Ann Surg Oncol Springer-Verlag. 2011;18: 2357-63.

18. Lodhia KA, Dale OT, Winter SC. Irrigation solutions in head and neck cancer surgery: a preclinical efficacy study. Ann Otol Rhinol Laryngol SAGE Publications. 2015;124:68-71.

19. Hah JH, Roh DH, Jung $\mathrm{YH}$, Kim KH, Sung M-W. Selection of irrigation fluid to eradicate free cancer cells during head and neck cancer surgery. Head neck. Wiley subscription services, Inc. A Wiley Company. 2012;34:546-50.

20. Zhou C, Ren Y, Li J, Li X, He J, Liu P. Systematic review and meta-analysis of rectal washout on risk of local recurrence for cancer. - PubMed - NCBI. J Surg Res. 2014;189:7-16.

21. Moskovitz B, Levin DR. Intravesical irrigation with distilled water during and immediately after transurethral resection and later for superficial bladder cancer. Eur Urol. 1987;13:7-9.

22. Sakai Y, Fujii Y, Hyochi N, Masuda H, Kawakami S, Kobayashi T, et al. A large amount of distilled water ineffective for prevention of bladder cancer cell implantation at the time of transurethral resection. Hinyokika Kiyo. 2006;52:173-5.

23. Amos S, Gofrit ON. Prevention of bladder tumor recurrence. Evolving trends in urology. In: Sashi S Kommu, editor. 1st ed. Rijeka, Croatia: InTech; 2012. pp. 69-76. https://doi.org/10.5772/38495.

24. Messing EM, Tangen CM, Lerner SP, Sahasrabudhe DM, Koppie TM, Wood DP, et al. Effect of Intravesical instillation of gemcitabine vs saline immediately following resection of suspected low-grade non-muscleinvasive bladder Cancer on tumor recurrence: SWOG S0337 randomized clinical trial. JAMA. 2018;319:1880-8.

Ready to submit your research? Choose BMC and benefit from:

- fast, convenient online submission

- thorough peer review by experienced researchers in your field

- rapid publication on acceptance

- support for research data, including large and complex data types

- gold Open Access which fosters wider collaboration and increased citations

- maximum visibility for your research: over $100 \mathrm{M}$ website views per year

At $\mathrm{BMC}$, research is always in progress.

Learn more biomedcentral.com/submissions 